\title{
Identification of Motive Forces on the Whole Body System during Walking
}

\author{
Raghdan J. AlKhoury, ${ }^{1}$ Suraj Joshi, ${ }^{1}$ Rama B. Bhat, ${ }^{1}$ and Shiping $\mathrm{Ma}^{2}$ \\ ${ }^{1}$ Department of Mechanical and Industrial Engineering, Concordia University, Sir George Williams Campus, \\ 1515 St. Catherine Street West, Montréal, QC, Canada H3G 2W1 \\ ${ }^{2}$ Department of Exercise Science, Concordia University, Loyola Campus, 7141 Sherbrooke Street West, Montréal, QC, Canada H4B 1R6
}

Correspondence should be addressed to Suraj Joshi, sjoshi@encs.concordia.ca

Received 28 June 2010; Accepted 13 September 2010

Academic Editor: K. M. Liew

Copyright ( $\odot 2010$ Raghdan J. AlKhoury et al. This is an open access article distributed under the Creative Commons Attribution License, which permits unrestricted use, distribution, and reproduction in any medium, provided the original work is properly cited.

Motive forces by muscles are applied to different parts of the human body in a periodic fashion when walking at a uniform rate. In this study, the whole human body is modeled as a multidegree of freedom (MDOF) system with seven degrees of freedom. In view of the changing contact conditions with the ground due to alternating feet movements, the system under study is considered piecewise time invariant for each half-period when one foot is in contact with the ground. Forces transmitted from the body to the ground while walking at a normal pace are experimentally measured and numerically simulated. Fourth-order RungeKutta method is employed to numerically simulate the forces acting on different masses of the body. An optimization problem is formulated with the squared difference between the measured and simulated forces transmitted to the ground as the objective function, and the motive forces on the body masses as the design variables to solve.

\section{Introduction}

A human body may essentially be considered a multidegree of freedom (MDOF) vibrating system. Whole body vibration studies and the related adverse health effects have been a topic of great interest and many researchers have attempted to model the parameters of MDOF models of the whole body and body parts [1-3]. Such models have been successfully employed in ride vibrations, ride comfort studies, and driver-seat dynamics [4-7]. Some researchers have studied comparative evaluation of different models of the same body part as well $[8,9]$. In these models, the mass information is rather simple; and the stiffness and damping values of the various elements in the MDOF system have been reported based on experimental studies $[1,10]$. However, there has virtually been no attempt to identify the muscular motive forces acting on different parts of the human body when the body itself performs simple physical activities instead of being excited by external sources.
Parameter identification can be carried out by subjecting the human body to sinusoidal excitation on a vibrating platform and curve fitting the analytical model results onto the experimental results $[11,12]$. However, such vibration excitation can cause undue reactions and discomfort to the body. Even though such a technique can be safely used on a healthy and normal individual, it cannot be used on subjects with poor health or with a medical condition for obvious reasons. For example, R. B. Bhat and P. P. Bhat [13] studied the dynamics of a foetus, uterus, and body model of a pregnant woman in order to study the dynamic force transmitted to the cervix from the foetus when the mother's body is subjected to ride vibrations. The parameters had to be chosen approximately from available information, as there is no way to obtain these parameters through vibration tests on the pregnant mother.

In this study, the body of a walking human is modeled as a 7 degrees of freedom system, taking into consideration the changing contact conditions due to the single foot contact 
with the ground during each half-period. Previous studies by Bhat [14] assumed that the sinusoidal excitation was exerted by the ground on the person while walking. McGibbon [15], Lewis and Ferris [16], and Garcia et al. [17] have chosen rather more simplistic models, with only two rigid links connected at the hip and some minor modifications of such model in order to simulate different methods of actuation. A major limitation of these simple bipedal models is that they cannot incorporate hip torques and forces. Furthermore, the contributions of other parts of the human body are completely disregarded in these models. In this study, the 7 DOF model employed does not incorporate any rotation around body joints and motion out of the saggittal plane. However, it does include different parts of the body modeled as simple spring-mass-damper systems. The excitation is considered as the sum total of self-excitation due to the change in the foot contact condition with the ground, the excitation by the muscles, and the joint reaction forces represented by the lumped force acting on each mass.

The force transmitted from the walking human to the ground is simulated in terms of motive forces applied by the muscles on different parts of the human body while walking, which is then curve fitted to the experimentally measured results using MATLAB [18] optimization module. Different motive forces are applied on different parts of the body since the masses, spring constants, and damping coefficients are all different owing to dissimilar morphological characteristics. The motive force defined in the paper does not only result from a specific muscle or muscles in the body, but is also a resultant force coming from active forces from muscles, and passive forces from ligaments, tendons and interactions between bones at joints called joint reaction forces. There are other models that relate motive forces to specific muscles in the body, but the model used in this paper does not consider the details of the anatomical structure of the muscles and other soft tissues. This paper presents the optimized approximations for these musculoskeletal motive forces using the method of least squares by matching the experimentally measured forces transmitted to the ground with simulated results.

\section{MDOF Model}

The 7 degrees of freedom model of a human body employed to study the walking dynamics is shown in Figure 1(a). Masses $m_{1}, m_{2}$ and $m_{3}$ represent the discretized head, trunk which includes the upper limbs, and the hip (or pelvis) parts, while $m_{4}$ and $m_{5}$ represent the thighs and $m_{6}$ and $m_{7}$ represent the legs including feet on right and left sides of the body, respectively. The $k_{i}^{\prime} s$ and $c_{i}^{\prime} s$ indicate the effective springs and dampers representing the viscoelastic characteristics of the muscles and joints. The springs and dampers $k_{7}, k_{8}, c_{7}$, and $c_{8}$ are varying as constant functions of time, depending on whether both right and left legs are in contact with the ground (double leg support) or one leg is in swing phase (single leg support). These stiffness and
TABLE 1: Values for masses, stiffness and damping coefficients.

\begin{tabular}{lcc}
\hline$m_{i}$ in $\mathrm{kg}$ & $k_{i} \times 10^{3}$ in N/m & $c_{i}$ in $\mathrm{N} \cdot \mathrm{s} / \mathrm{m}$ \\
\hline$m_{1}=5.5$ & $k_{1}=310$ & $c_{1}=400$ \\
$m_{2}=20$ & $k_{2}=183$ & $c_{2}=4750$ \\
$m_{3}=25$ & $k_{3}=162.8$ & $c_{3}=4585$ \\
$m_{4}=9$ & $k_{4}=162.8$ & $c_{4}=4585$ \\
$m_{5}=9$ & $k_{5}=162.8$ & $c_{5}=2064$ \\
$m_{6}=6$ & $k_{6}=162.8$ & $c_{6}=2064$ \\
$m_{7}=6$ & $k_{7}=162.8$ & $c_{7}=2064$ \\
& $k_{8}=162.8$ & $c_{8}=2064$ \\
& $k_{9}=162.8$ & $c_{9}=2064$ \\
& $k_{10}=162.8$ & $c_{10}=2064$ \\
\hline
\end{tabular}

damping coefficients in different time periods of a gait cycle are considered as follows:

$k_{7} \neq 0, \quad c_{7} \neq 0, \quad k_{8} \neq 0, \quad c_{8} \neq 0, \quad$ in double leg support,

$k_{7} \neq 0, \quad c_{7} \neq 0, \quad k_{8}=0, \quad c_{8}=0, \quad$ in right single support,

$k_{7}=0, \quad c_{7}=0, \quad k_{8} \neq 0, \quad c_{8} \neq 0, \quad$ in left single support,

Figure 1(b) shows the free body diagram of a representative mass $m_{7}$. The equation of motion for the MDOF whole body system is written as

$$
[M]\{\ddot{x}\}+[C(t)]\{\dot{x}\}+[K(t)]\{x\}=\left\{F_{g}\right\}+\left\{F_{m}(t)\right\},
$$

where $\left\{F_{g}\right\}$ and $\left\{F_{m}(t)\right\}$ are the gravity and muscular forces, respectively. Gravity forces are easily computed as the weight of each of the masses. Most of the stiffness and damping coefficients are taken from published literature [14], and reasonable values are assumed for the rest. These are presented in Table 1. The undamped natural frequencies (i.e., square roots of eigen values) and corresponding normal modes (eigenvectors) for this 7 DOF model are presented in Table 2 from a MATLAB run.

\section{Experimental Measurements}

Ground reaction forces (GRF's) are measured when a human subject walks on force platforms fitted with transducers. During the experiment, the subject walks at a steady pace towards the force platforms for a considerable distance in order to achieve steady state walking conditions. These measured GRF's can thus be reasonably assumed to be the forces transmitted from the human body to the ground under steady state walking conditions. As the time period in double leg support during walking is very short, it is assumed that only one foot is in contact with the ground at any time and hence the full body weight will be carried by the right leg or the left leg for a half period in a gait cycle. In this experiment, the subject stepped on a set of four force platforms in steady state walking condition, which were arranged in a square pattern as shown in Figure 2. The forces transmitted by the right and the left feet contacting 
TABLE 2: Natural frequencies and normal modes.

\begin{tabular}{lccccccc}
\hline Natural frequencies $(\mathrm{Hz})$ & 2.07 & 3.78 & 4.47 & 5.25 & 6.14 & 6.49 & 6.59 \\
\hline \multirow{3}{*}{ Normal modes } & 1.00 & 0.65 & -0.43 & 0.35 & -0.11 & 0.16 & -1.00 \\
& 0.99 & 0.56 & -0.31 & 0.16 & 0.00 & -0.04 & 0.32 \\
& 0.89 & -0.09 & 0.43 & -0.69 & 0.18 & -0.08 & -0.06 \\
& 0.61 & -0.09 & 1.00 & 0.90 & 0.01 & 0.58 & 0.07 \\
& 0.95 & -0.70 & -0.34 & -0.07 & -1.00 & 0.06 & 0.04 \\
& 0.31 & -0.06 & 0.70 & 1.00 & -0.19 & -1.00 & -0.10 \\
& 0.98 & -1.00 & -0.82 & 0.67 & 0.93 & -0.04 & -0.02 \\
\hline
\end{tabular}

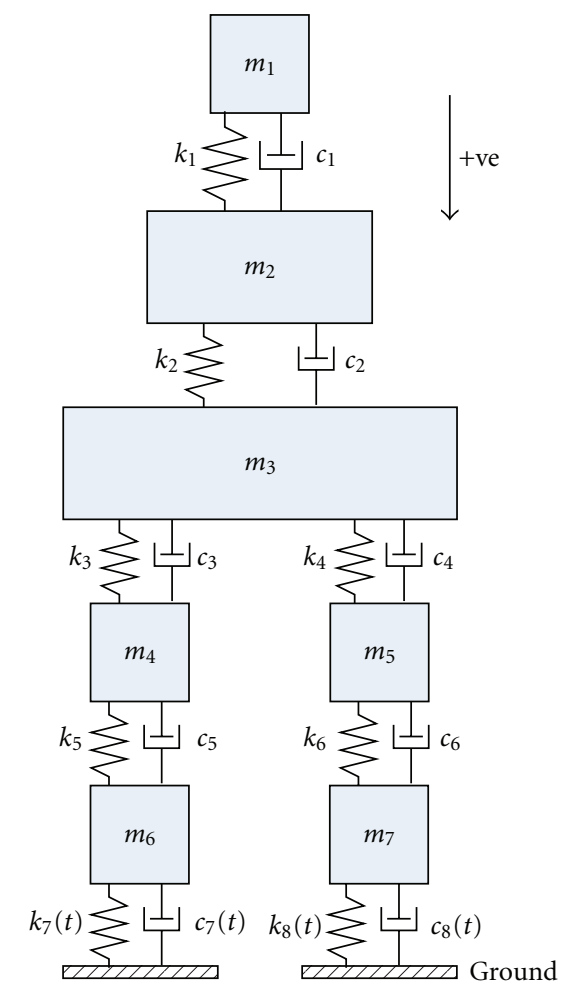

(a)

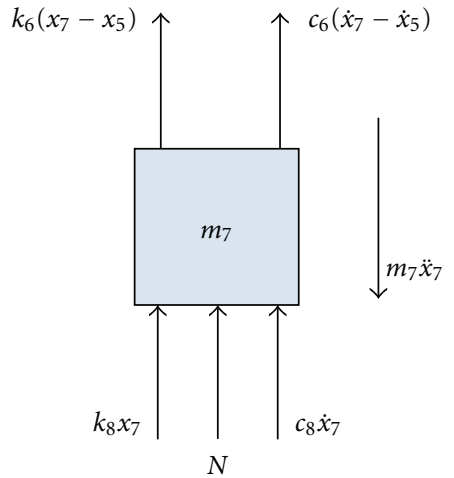

(b)

Figure 1: (a) 7 DOF model of the human body, and (b) Free body diagram of $m_{7}$.
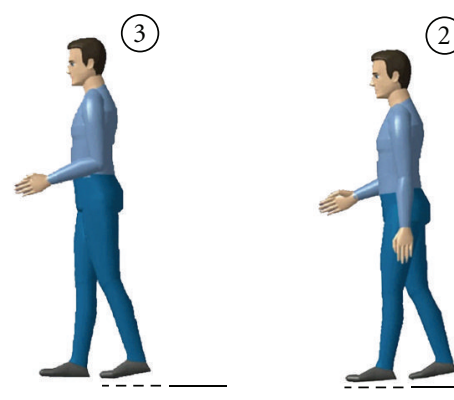

(2)

Layout of force platforms

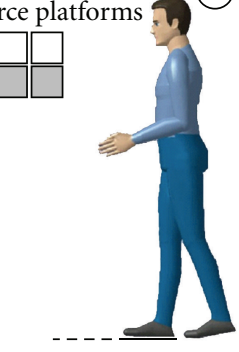

Direction of motion

FIGURE 2: Experiment scheme.

in succession were measured on two force platforms. At the instant of peak transmissibility, the whole body would be standing on only one foot, with the other being entirely in air; however, the measured force transmitted to the force platform would be slightly higher than the observed body weight of the subject owing to the dynamic effects of the body.

Figure 3 shows the results of measurements in one typical run of the experiment. The first peak in the measured signal indicates the force exerted on the platform when the heel hits the platform. The second peak, which is slightly lower than the first, indicates the corresponding force exerted when the toe hits the platform and the heel begins to rise. The trough in between the two peaks is indicative of the straddling motion of the sole between the two contacts. It should also be noted that the signals for the right and the left legs overlap slightly over time due to the fact that the heel of the second foot would have already hit the platform in the front while 


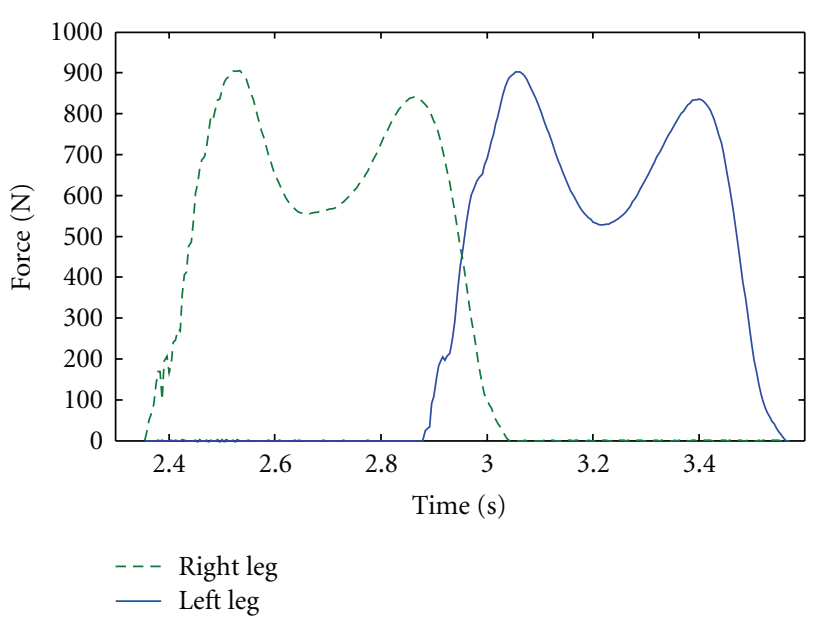

FIgURE 3: Transmitted forces in one typical run of experiment.

the toes of the first foot leave contact with the platform behind. The forces exerted by the two feet on the force platforms were measured for a period of approximately $5 \mathrm{~s}$, which is the time required to complete one cycle of walk on the two platforms in line.

\section{Formulation and Optimization}

The forces transmitted to the ground are expressed as

$$
\begin{aligned}
& T_{1}=k_{7}(t) x_{6}(t)+c_{7}(t) \dot{x}_{6}(t), \\
& T_{2}=k_{8}(t) x_{7}(t)+c_{8}(t) \dot{x}_{7}(t),
\end{aligned}
$$

where $T_{1}$ and $T_{2}$ represent the forces transmitted through the right and the left feet, respectively.

Casting the equations of motion in the state-space form, fourth-order Runge-Kutta integration is carried out in MATLAB in order to obtain the responses and forces transmitted to the ground. The set of forces $\left\{F_{m}(t)\right\}$ are identified as design variables, and the squared error between the measured and computed forces transmitted to the ground is the objective function to be minimized. During walking the human upper body is usually maintained in a neutral position in which the muscle activities in the upper body for maintaining the posture are trivial compared to the muscle activity in the lower extremities [19]. Therefore, it is assumed in this study that the muscular forces acting on the head mass $\left(m_{1}\right)$ and the trunk mass $\left(m_{2}\right)$ are zero during walking. The forces at all the other degrees of freedom are periodic. It is also assumed that the forces on the degrees of freedom 4 and 6 are identical to those on 5 and 7, respectively, with a phase shift of $180^{\circ}$. This is because an ideal human body is completely symmetric along the vertical midline, and one half-side can be taken as an exact mirror image of the other.

From Figure 3 it can be seen that one complete period for the right and left foot contact in succession is $1.2167 \mathrm{~s}$ and the transmissibility consists of four peaks corresponding to the successive right and left heel and the toe contact to the ground. Assuming that the four peaks are caused by four force pulses, the forces $\left\{F_{m}(t)\right\}$ are taken as

$$
\left\{F_{m}(t)\right\}=\left\{A_{1}\right\} f_{1}(t)+\left\{A_{2}\right\} f_{2}(t)+\left\{A_{3}\right\} f_{3}(t)+\left\{A_{4}\right\} f_{4}(t),
$$

where $f_{i}(t), i=1,2,3$, and 4 are predefined pulse forms and their amplitudes are given by the vectors $\left\{A_{i}\right\}$. In general $f_{i}(t)$ can be of different durations within the period, but in this study they are assumed to be of equal durations and are rectangular, as shown in Figure 4. They are further expanded in Fourier series in order to assign smooth, varying sinusoidal waveforms for the force pulses $f_{i}(t)$ as below:

$$
\begin{aligned}
& f_{1}=a_{1,0}+\sum_{n=1}^{N}\left(a_{1, n} \cos \left(\frac{2 n \pi t}{T}\right)+b_{1, n} \sin \left(\frac{2 n \pi t}{T}\right)\right), \\
& f_{2}=a_{2,0}+\sum_{n=1}^{N}\left(a_{2, n} \cos \left(\frac{2 n \pi t}{T}\right)+b_{2, n} \sin \left(\frac{2 n \pi t}{T}\right)\right), \\
& f_{3}=a_{3,0}+\sum_{n=1}^{N}\left(a_{3, n} \cos \left(\frac{2 n \pi t}{T}\right)+b_{3, n} \sin \left(\frac{2 n \pi t}{T}\right)\right), \\
& f_{4}=a_{4,0}+\sum_{n=1}^{N}\left(a_{4, n} \cos \left(\frac{2 n \pi t}{T}\right)+b_{4, n} \sin \left(\frac{2 n \pi t}{T}\right)\right) .
\end{aligned}
$$
follows:

Using (4), the muscular motive forces can be obtained as

$$
\begin{gathered}
\left\{F_{m}(t)\right\}=\left\{\alpha_{0}\right\}+\left\{\sum_{n=1}^{N}\left(\beta_{n} \cos \left(\frac{2 n \pi t}{T}\right)+\gamma_{n} \sin \left(\frac{2 n \pi t}{T}\right)\right)\right\}, \\
\text { where }\left\{\alpha_{0}\right\}=\left\{A_{1}\right\} a_{1,0}+\left\{A_{2}\right\} a_{2,0}+\left\{A_{3}\right\} a_{3,0}+\left\{A_{4}\right\} a_{4,0}, \\
\left\{\beta_{n}\right\}=\left\{A_{1}\right\} a_{1, n}+\left\{A_{2}\right\} a_{2, n}+\left\{A_{3}\right\} a_{3, n}+\left\{A_{4}\right\} a_{4, n}, \\
\left\{\gamma_{n}\right\}=\left\{A_{1}\right\} b_{1, n}+\left\{A_{2}\right\} b_{2, n}+\left\{A_{3}\right\} b_{3, n}+\left\{A_{4}\right\} b_{4, n} .
\end{gathered}
$$

The resulting four shapes, $f_{i}(t), i=1,2,3$, and 4 are shown in Figure 5.

Nonlinear iterative least square curve fitting from MATLAB [18] optimization toolbox is used, and the error between the measured and computed transmitted forces to the ground is calculated using the following equation:

$$
E=\frac{1}{2} \sum_{i=1}^{N}\left(T_{1, i}\left(\left[\left\{A_{1}\right\}^{T},\left\{A_{2}\right\}^{T},\left\{A_{3}\right\}^{T},\left\{A_{4}\right\}^{T}\right]\right)-T_{\text {exp }, i}\right)^{2},
$$

where $N$ is the size of the vector $\left\{T_{1}\right\}$ and $\left\{T_{\exp }\right\}$, and the notations $\left\{T_{1, i}\right\}$ and $\left\{T_{\exp , i}\right\}$ stand for the $i$ th element of the vectors $\left\{T_{1}\right\}$ and $\left\{T_{\exp }\right\}$, respectively. Note that $\left\{T_{1}\right\}$ is a function of $\left\{A_{1}\right\},\left\{A_{2}\right\},\left\{A_{3}\right\}$, and $\left\{A_{4}\right\}$ and is not multiplied by them.

Figure 6 shows a workflow schematic of the numerical integration and optimization process. Only $T_{1}$ is considered in numerical computation assuming the symmetrical nature of the problem as indicated before. 


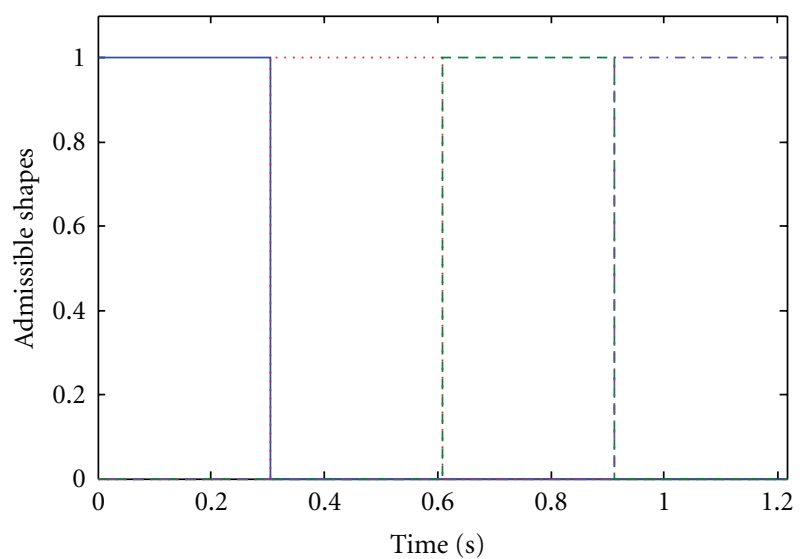

FIgURE 4: Admissible unit force functions.

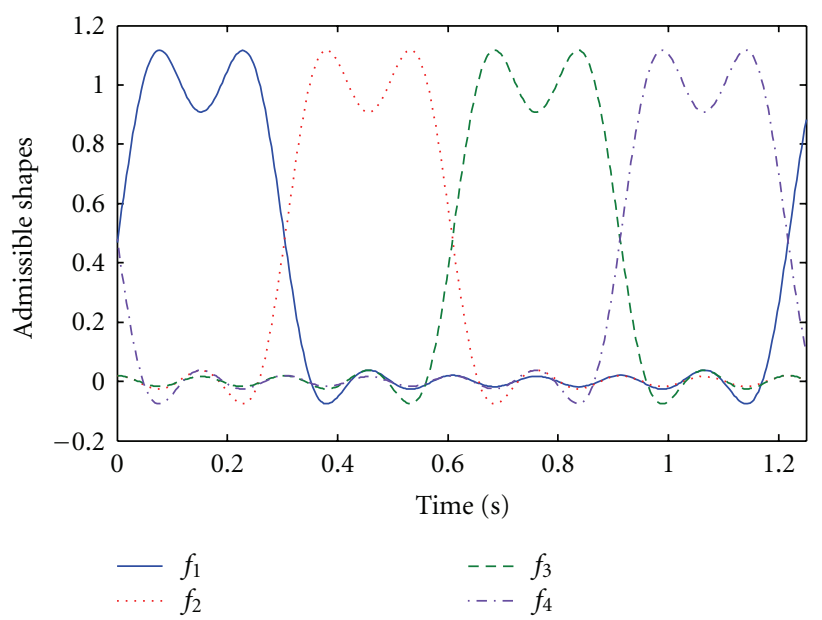

Figure 5: Generalized shapes of admissible force functions.

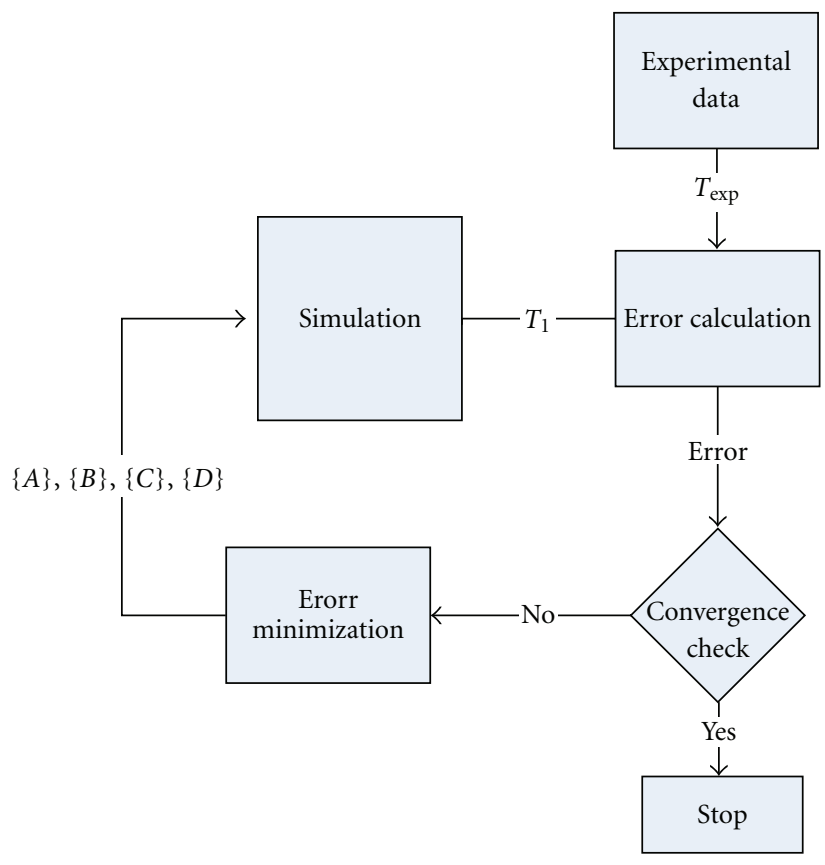

Figure 6: Optimization process.

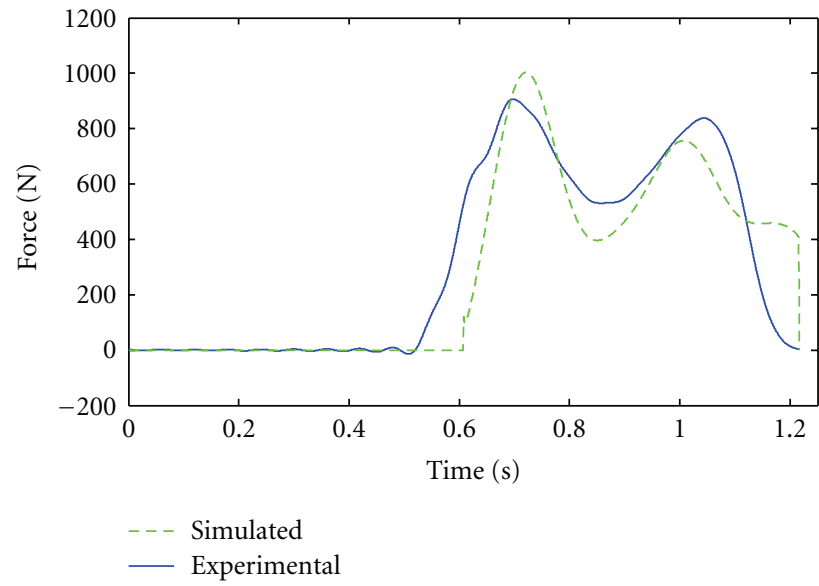

Figure 7: Experimental and simulated transmitted forces.

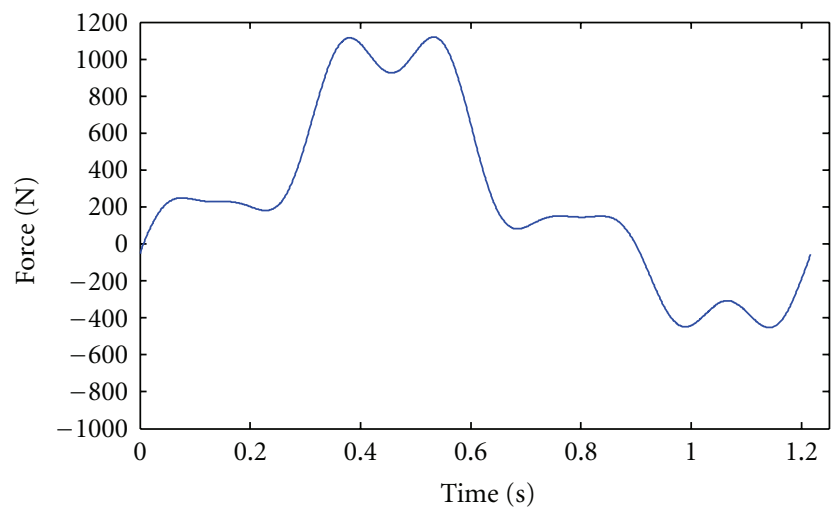

FIgURE 8: $\left\{F_{3}(t)\right\}$ force profile over one time period.

\section{Results and Discussion}

Least squares curve fitting resulted in a set of coefficients that indicated the transmitted forces as shown in Figure 7. The measured forces transmitted to the ground compare closely with those obtained by simulation.

Figure 8 shows the force profile through one period of the force $\left\{F_{3}(t)\right\}$ acting on the hip. Figure 9 shows the plots of $\left\{F_{4}(t)\right\}$ and $\left\{F_{5}(t)\right\}$, and Figure 10 shows those of $\left\{F_{6}(t)\right\}$ and $\left\{F_{7}(t)\right\}$. It can be seen from both figures that these forces are similar with a shift of one half-period, which is originally set as a constraint in the simulation. Moreover, Figure 10 shows that during the foot contact, precisely represented from time $0.6 \mathrm{~s}$ to $1.2 \mathrm{~s}$ of $\left\{F_{6}(t)\right\}$ corresponding to the step involving the spring and damper $k_{7}$ and $c_{7}$, the lumped muscular force acting on the mass $m_{6}$ is positive when the heel is landing and the load is all directed to the ground. In the next half-period, the muscle force applied to the foot is lifting the whole body to move upward. This force is almost equal to the weight of the body.

Comparing the force profiles in Figures 8, 9, and 10, $\left\{F_{3}(t)\right\}$ shows the highest overall value. This result is in agreement with previous observations reported in other published literature $[16,17,20,21]$, that the force acting 


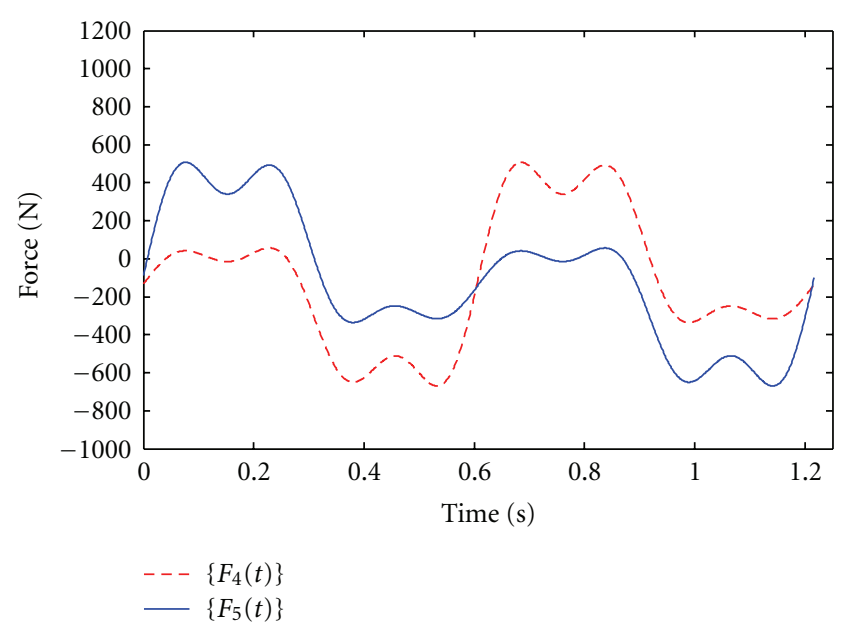

FIGURE 9: $\left\{F_{4}(t)\right\}$ and $\left\{F_{5}(t)\right\}$ force profiles over one time period.

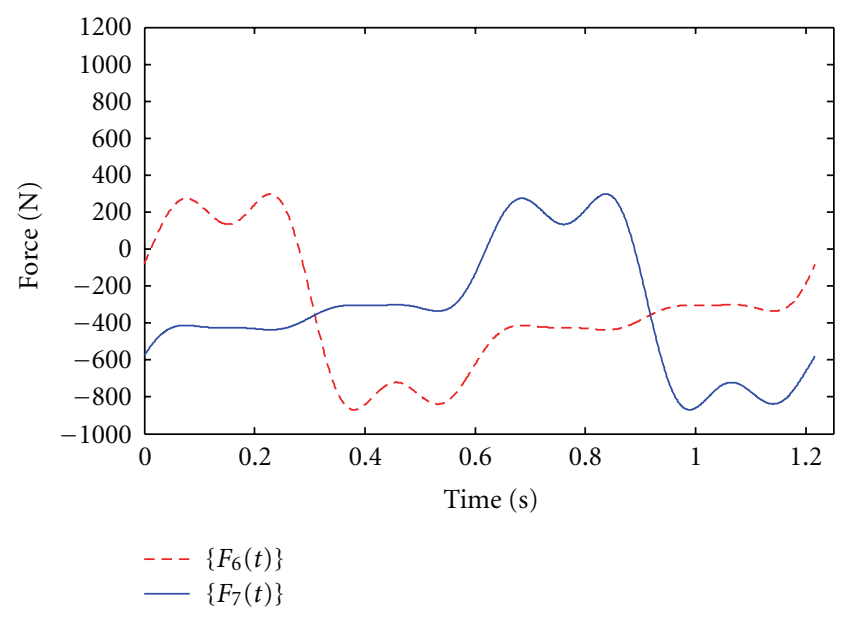

FIGURE 10: $\left\{F_{6}(t)\right\}$ and $\left\{F_{7}(t)\right\}$ force profiles over one time period.

on the hips is quite significant in comparison to that acting on the thighs and legs during walking or running activity. This significant force is assumedly due to the greatly increased muscle contraction of the hip abductor muscles for maintaining the upper body in a vertical position during walking with a single leg support.

The implications of this conclusion are quite significant to those suffering from musculoskeletal disorders, osteoarthritis, or diabetes in advanced stages, especially in the hips and the lower limbs. People suffering from joint pains in the lower limbs may use a less tiring mechanism with smaller step length during walking. Conversely, people with hip pain can walk with an exaggerated lower limb movement. Such recommendations have also been made by Mueller et al. [21] and Lewis and Sahrmann [22]. Based on the reason proposed above, a walking pattern in which a small lateral bending of upper body to the ipsilateral side of the supporting leg during walking might be able to reduce the hip abductor muscle contraction so that the load applied to the hip joint could be significantly reduced. This assumption can be investigated in further studies with this 7 DOF model. The effect of arm swing on ground reaction forces in gait also contributes substantially to the vertical ground reaction forces, and an 8 or 9 DOF model with the arms separated from the trunk could be a better representation for including the arm swing effect $[23,24]$.

The 7 DOF model incorporates contributions of different parts of the body on force distributions in the 5 relevant degrees of freedom that are involved in the physical process of walking. While such a detailed model has rarely been studied hitherto in gait studies, a major limitation of this 7 DOF model is that it considers the motion of hips, thighs, and legs only in the vertical direction in the saggittal plane. This simplified 7 DOF model also tends to ignore the rotational movements of different body parts around their joints while walking. The upper body parts and lower limbs may also have small movements laterally in the frontal plane, which will affect the muscular forces in the vertical direction. Furthermore, the relative movement between the legs and feet is not incorporated as separate masses with additional DOF's in this model.

In the normal walking process, the arms usually swing back and forth which would contribute to the inertia forces of the body. However, in this model the upper limbs are incorporated into the trunk mass $\left(m_{2}\right)$ and their movements with respect to the trunk during walking are completely ignored. The effect of this simplification on inertia force of arms depends on the degree of arm swinging. In this study, the simplification was justified by the fact that the degree of arm swinging was found small and the effect of inertia force induced by the arm movement in vertical direction could be largely mitigated.

It is noted that all the degrees of freedom in this model are taken as lumped masses, as it is difficult to come up with an exact formulation of distributed mass system of different body parts. Such data is largely non-existent in biomedical literature, and moreover, a universal distributed mass system is extremely difficult to formulate owing to different musculoskeletal characteristics of individual people. This model is at best simply a rough approximation of relevant parts of the human body with a focus on predicting and comparing force distributions in chosen discretized masses while walking at a normal pace and with a normal gait.

\section{Conclusions}

Experiments were carried out to measure forces transmitted to force platforms when a human subject is walking. The human body is modeled as a 7 degrees of freedom system. The forces transmitted to the ground are measured and the muscle forces acting on the masses are estimated by fitting least squares curve to the measured forces transmitted to the ground. The results show that the force acting on the hip is quite significant in comparison to forces acting on the thighs and legs. Relevant conclusions are drawn about the suitability of this 7 DOF model and the implications of these observations for people with musculoskeletal disabilities or ailments. 


\section{References}

[1] J. H. Dong, R. G. Dong, S. Rakheja, D. E. Welcome, T. W. McDowell, and J. Z. Wu, "A method for analyzing absorbed power distribution in the hand and arm substructures when operating vibrating tools," Journal of Sound and Vibration, vol. 311, no. 3-5, pp. 1286-1304, 2008.

[2] International Standard ISO 2631-1: 1997/Amd 1:2000 (E). Mechanical vibration and shock-evaluation of human exposure to whole body vibration-part 1: general requirements; Amendment 1.

[3] X. Xie, Absorbed power as a measure of whole body vehicular vibration exposure, M.S. thesis, Department of Mechanical and Industrial Engineering, Concordia University, Montréal, Canada, 2001.

[4] N. Nawayseh and M. J. Griffin, "A model of the vertical apparent mass and the fore-and-aft cross-axis apparent mass of the human body during vertical whole-body vibration," Journal of Sound and Vibration, vol. 319, no. 1-2, pp. 719-730, 2009.

[5] A. G. Mayton, N. K. Kittusamy, D. H. Ambrose, C. C. Jobes, and M. L. Legault, "Jarring/jolting exposure and musculoskeletal symptoms among farm equipment operators," International Journal of Industrial Ergonomics, vol. 38, no. 910, pp. 758-766, 2008.

[6] Y. Yang, W. Ren, L. Chen, M. Jiang, and Y. Yang, "Study on ride comfort of tractor with tandem suspension based on multibody system dynamics," Applied Mathematical Modelling, vol. 33, no. 1, pp. 11-33, 2009.

[7] W. Wang, S. Rakheja, and P.-É. Boileau, "Relationship between measured apparent mass and seat-to-head transmissibility responses of seated occupants exposed to vertical vibration," Journal of Sound and Vibration, vol. 314, no. 3-5, pp. 907-922, 2008.

[8] S. Rakheja, J. Z. Wu, R. G. Dong, A. W. Schopper, and P.-É. Boileau, "A comparison of biodynamic models of the human hand-arm system for applications to hand-held power tools," Journal of Sound and Vibration, vol. 249, no. 1, pp. 55-82, 2002.

[9] D. D. Reynolds and E. N. Angevine, "Hand-arm vibration, part II: vibration transmission characteristics of the hand and arm," Journal of Sound and Vibration, vol. 51, no. 2, pp. 255265, 1977.

[10] R. G. Dong, A. W. Schopper, T. W. McDowell et al., "Vibration energy absorption (VEA) in human fingers-handarm system," Medical Engineering \& Physics, vol. 26, no. 6, pp. 483-492, 2004.

[11] B. Bazrgari, A. Shirazi-Adl, and M. Kasra, "Computation of trunk muscle forces, spinal loads and stability in whole-body vibration," Journal of Sound and Vibration, vol. 318, no. 4-5, pp. 1334-1347, 2008.

[12] A. Godfrey, R. Conway, D. Meagher, and G. ÓLaighin, "Direct measurement of human movement by accelerometry," Medical Engineering and Physics, vol. 30, no. 10, pp. 1364-1386, 2008.

[13] R. B. Bhat and P. P. Bhat, "Biomechanical considerations in antenatal care of high risk pregnancies," in Proceedings of the 18th Biennial Conference on Mechanical Vibration and Noise, pp. 911-915, Pittsburgh, PA, USA, September 2001.

[14] R. B. Bhat, "Dynamic response of whole body system subjected to walking generated excitation," in Proceedings of the 19th ASME Biennial Conference on Mechanical Vibration and Noise (VIB '03), vol. 5, pp. 1555-1558, Chicago, IL, USA, 2003.
[15] C. A. McGibbon, "Toward a better understanding of gait changes with age and disablement: neuromuscular adaptation," Exercise and Sport Sciences Reviews, vol. 31, no. 2, pp. 102-108, 2003.

[16] C. L. Lewis and D. P. Ferris, "Walking with increased ankle pushoff decreases hip muscle moments," Journal of Biomechanics, vol. 41, no. 10, pp. 2082-2089, 2008.

[17] M. Garcia, A. Chatterjee, A. Ruina, and M. Coleman, "The simplest walking model: stability, complexity, and scaling," Journal of Biomechanical Engineering, vol. 120, no. 2, pp. 281286, 1998.

[18] F. Gustafsson and N. Bergman, MATLAB for Engineers Explained, Springer, New York, NY, USA, 2003.

[19] E. Asmussen and K. Klausen, "Form and function of the erect human spine," Clinical Orthopaedics, vol. 25, pp. 55-63, 1962.

[20] M. J. Mueller, S. D. Minor, S. A. Sahrmann, J. A. Schaaf, and M. J. Strube, "Differences in the gait characteristics of patients with diabetes and peripheral neuropathy compared with agematched controls," Physical Therapy, vol. 74, no. 4, pp. 299 313, 1994.

[21] M. J. Mueller, D. R. Sinacore, S. Hoogstrate, and L. Daly, "Hip and ankle walking strategies: effect on peak plantar pressures and implications for neuropathic ulceration," Archives of Physical Medicine and Rehabilitation, vol. 75, no. 11, pp. 11961200, 1994.

[22] C. L. Lewis and S. A. Sahrmann, "Acetabular labral tears," Physical Therapy, vol. 86, no. 1, pp. 110-121, 2006.

[23] S. H. Collins, P. G. Adamczyk, and A. D. Kuo, "Dynamic arm swinging in human walking," Proceedings of the Royal Society $B$, vol. 276, no. 1673, pp. 3679-3688, 2009.

[24] R. Kram, A. Domingo, and D. P. Ferris, "Effect of reduced gravity on the preferred walk-run transition speed," Journal of Experimental Biology, vol. 200, no. 4, pp. 821-826, 1997. 

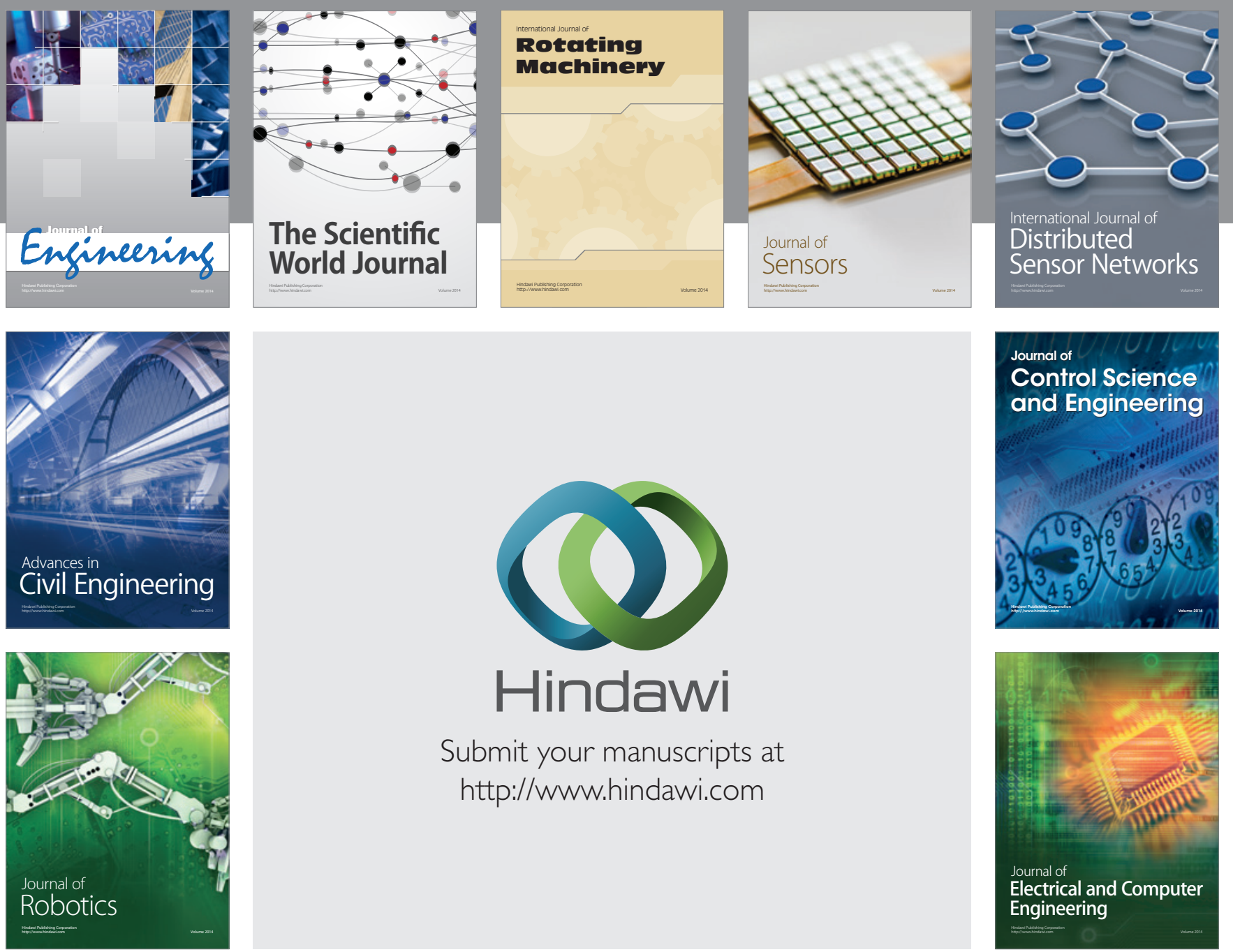

Submit your manuscripts at

http://www.hindawi.com
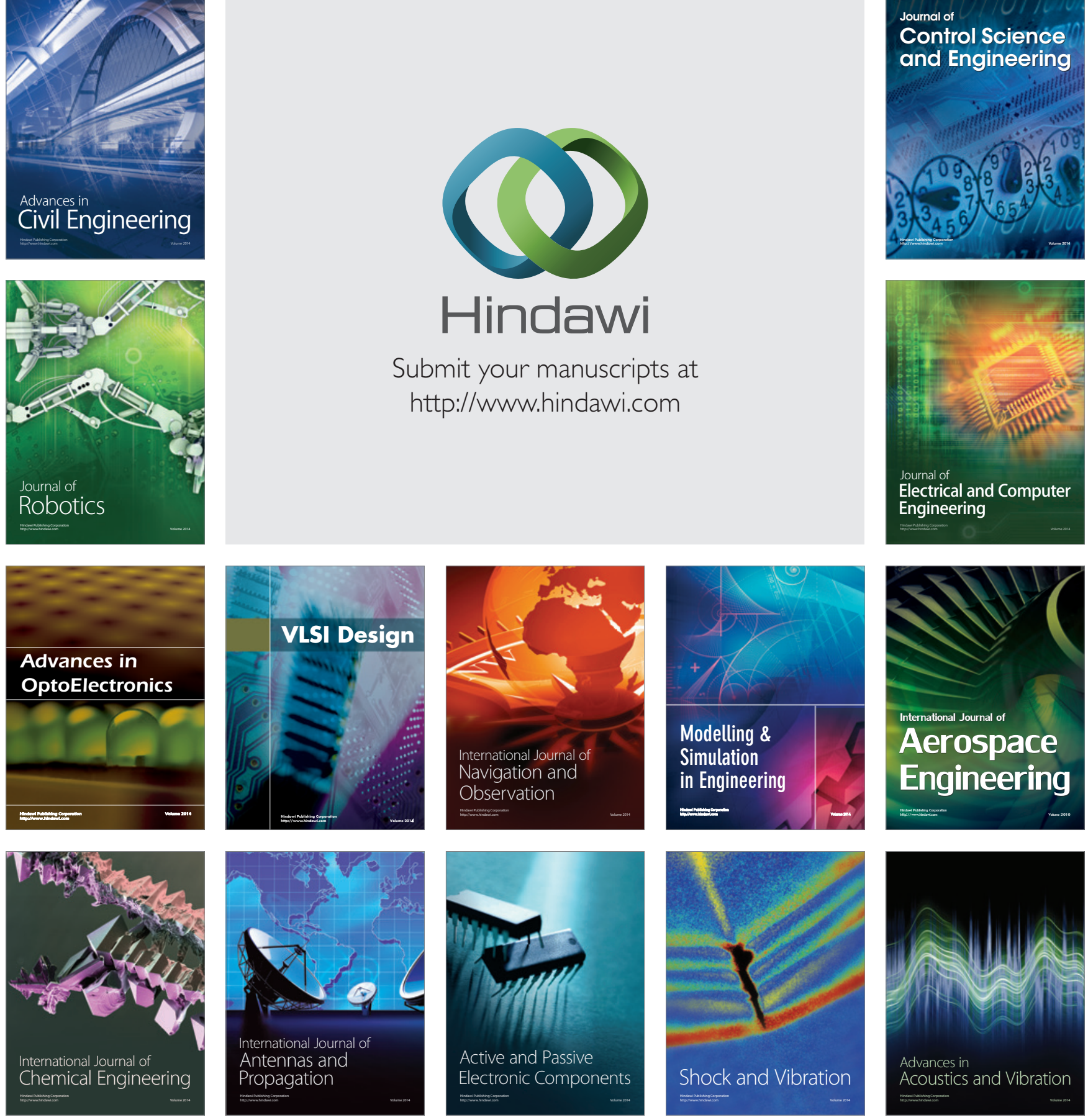